\title{
Development of a Process Based Data Driven Engineering Design Knowledge Reuse System
}

\author{
${ }^{1}$ David Baxter, ${ }^{2}$ James Gao \\ ${ }^{1}$ Cranfield University, d.baxter@cranfield.ac.uk \\ ${ }^{2}$ Cranfield University, james.gao@cranfield.ac.uk
}

\begin{abstract}
This paper will describe the development of the web enabled version of a process based engineering design knowledge reuse system. The rationale for using the design process as a central element of knowledge management will be discussed. The system structure will be described. Evaluation of the prototype showed the most valuable attributes of system. Mapping the design process helped to create the product data model. Workshops were used to validate the system. A small number of product parameters are required for developing the product concept in the early stages. The research showed the importance of multi view validation and iteration in system development. It also showed the importance of graphics in design support. Key issues include: the importance of process capture, data model validation, the use of graphics in the interface, system design and system assessment.
\end{abstract}

Keywords: Design Knowledge Management, Design Reuse, Process Representation

\section{INTRODUCTION}

The need to manage knowledge in the product development environment is driven by the need to reduce development time and achieve faster time to market. Global competitive pressures are increasing the importance of time to market in terms of lost sales and loss of market share through competitors locking in customers. Faster development also results in lower project costs: less design effort to finance, and lower cost of finance due to a shorter borrowing period. Cost pressures have also increased the workload on engineers and designers, so support tools that enable the reuse of design knowledge help the project team achieve right first time. Automation in the design process can also save time and improve reliability. Tools to provide visibility and communicate project progress enable distributed collaboration.

One approach to support the design effort is design reuse. There have been several methods proposed to support design reuse [1]. Design reuse is considered beneficial, however its application is problematic [2]. Computer aided design support methods have been investigated, and current gaps include function, design for manufacture, assembly, sustainability: "Design for $\mathrm{x}$ " (DFx), cost, requirements, design process, design intent, history (information archiving and retrieval), communication, and guidance [3]. Function, DFx, and cost are not addressed directly by the proposed system. Requirements are addressed, as the input to the system [4]. The content of the requirements specification is kept to a minimum, to reduce constraints on the design and as recognition for requirement generation during design having a much greater influence [5]. Design process support is integral. Intent is supported through information provision, which also aims to meet the design history need. The combination of these system elements represents design reuse. Communication (of current progress) is achieved through the process model and data model. Guidance is also achieved through the information provision.

The system described in this paper aims to support the effort to reduce time by providing enhanced design support in four categories: process support, data provision, project tracking, and knowledge based support. These issues will be described in turn, following a discussion showing the value of using the design process as a central element of knowledge management. Some examples of the methods applied to each of these categories in the system prototype will then be described. Next, a description of system interface development, then a discussion relating to evaluation of the system. Finally conclusions will be drawn.

\section{DESIGN PROCESS FOR KNOWLEDGE MANAGEMENT}


Within engineering design, the process follows roughly the same four steps: concept, preliminary, embodiment and detail $[6,7]$. The continued development of knowledge based support is reducing the number of steps required [8]. The design of similar products, such as new members of a product family (product variants) provides greater opportunity for design reuse than new or innovative design. Variant designs also have a development path that is more similar than for innovative design [9]. It is therefore possible to create a project model that describes the development process for a given project in a level of detail that directly corresponds to similarity to past products. The 'best practice' method for developing a similar product can be described to a high level of detail in advance, so it follows that a reuse method that applies the design process can include a high degree of reusable content. Designers must seek to reuse knowledge in the context of the design problem [10], and a project model can provide that context.

Where the product development process product technology are evolving, such as in new technology areas, a different approach is required. Design process support was one of the needs identified by Ullman [3]. The main issue was what task should be performed next. Clarkson and Hamilton developed the 'signposting' system in response to this need [11]. In a project that set out to support design reuse, the researchers required a model of the design process to map the knowledge requirements. There was no formally defined design process model, and the process capture effort uncovered the underlying importance of certainty in defining the next best step. The assumption made supporting the chosen model for this system is that the process (or those elements of the process which are mapped in detail) is relatively stable, with known technologies and so a known development path. Where new technologies or uncertain design elements are introduced, the process precedence model must be dynamically managed.

The following sections describe the research method that led to the current version of the system, followed by a description of the system elements that provide support to the process model. The research project aim is to propose a method that promotes and supports design knowledge reuse in mature domains. The companies collaborating on the research project are all working on highly engineered mature technology. The initial investigation showed that there was a strong interest in design reuse. The existing Knowledge Management capability varied within each of the companies, and so expectations also varied. The proposal had to be suitable to each company.

\section{REQUIREMENTS OF THE SYSTEM}

The research aim was to provide a method to enable the reuse of engineering design knowledge. The strategy adopted in order to place the research in context and create a test case was to focus on a single component of a representative product. Once the component was selected, the next step was to capture knowledge about that component that could be represented for reuse in future projects. This phase began with semi structured interviews, in which designers and engineers were asked:

- What elements of the component were important

- How the component interacted with other parts of the product

- What functions the component performed

- The approach taken to the design of the component

It became apparent through the analysis of the interview data that a small set of parameters were critical to the design of the component. The source of the parameters was also important, particularly with regard to whether the parameter is specified directly by the customer or if it was derived from an internal source. There were also a number of parameters relating to component fit that were required, along with certain standard parts and fixings. Given this rich context, the design of the component should be a straightforward task. Simply provide the necessary parameters and the optimum solution should be easy to achieve. This is not the case: the elements that were missing included relationships which were not well understood. This includes evaluations of performance and functionality which are based on judgement and expertise. Those relationships that are well understood, such as performance analysis of the vacuum pump, can be codified in analysis tools and knowledge based engineering systems. Those relationships which are not well understood, which will be omitted for commercial reasons, rely on the expertise of the design team to specify and evaluate the product. With this background, the following requirements were generated:

- $\quad$ Process support

- Data provision

- $\quad$ Project tracking

- Knowledge based support 
Process support includes task selection as well as task support: information provision for the current task. An element of this is data provision: making relevant product parameters available within the task. Project tracking requires data capture: storing design parameters once they are defined. Project tracking also includes workflow management: noting task completion and assigning new tasks. Knowledge based support includes calculations made on the data, which represent full or partial process automation. In the development of this system, only basic knowledge based support was demonstrated. It is assumed that existing KBE methods can be built into the framework, and become an integral (automated) part of the design process.

\section{SYSTEM DEVELOPMENT}

With the requirements of the system defined, the second stage of the research took place. This included the development of a design process model. The process was captured and represented using IDEFØ.

\subsection{System features: Process Support}

This design process modelling exercise highlighted some important factors:

- Modelling the process is itself a useful (knowledge management) activity

- The process representation method influences the process logic

- There are only a few key product parameters that are required for concept design

- The source of design parameters is an important factor in design decisions

The existing company capability was analysed alongside to contributions from various literature sources in design synthesis methods, design management, design automation, knowledge management and KBE. When these sources were combined with research relating to the applicability of a concurrent process [9], the conclusion was drawn that within a well known domain, a detailed design process model could be applied as a central element of the engineering design knowledge reuse system.

With the design process model completed, it became apparent that the IDEFØ representation could not easily be interpreted by the users performing validation and analysis tasks. The Design Roadmap (DR) method [12] was selected in place of IDEFØ. The data handling capability and simple representation of the DR method were the main factors influencing the choice. Additional capabilities, including an extensive range of link types and alternative representations, are not being used during this developmental stage. IDEFØ was also rejected as it was considered to be a major constraint on the modelling process: its rules and syntax added to the complexity of process capture, modelling, representation and understanding.

A partial process model is shown in

Figure 1. This shows the basic elements of the DR method, including tasks (in black) and features (in blue, round edges). The DR framework has two major elements: tasks and features. The feature is the data set created by a task. Each task has at least one feature as an input. The task node represents the overall task required. In the process model below the tasks are in the early design stages, from which the design requirements are specified and product parameters generated based on a mathematical model of the vacuum pump.

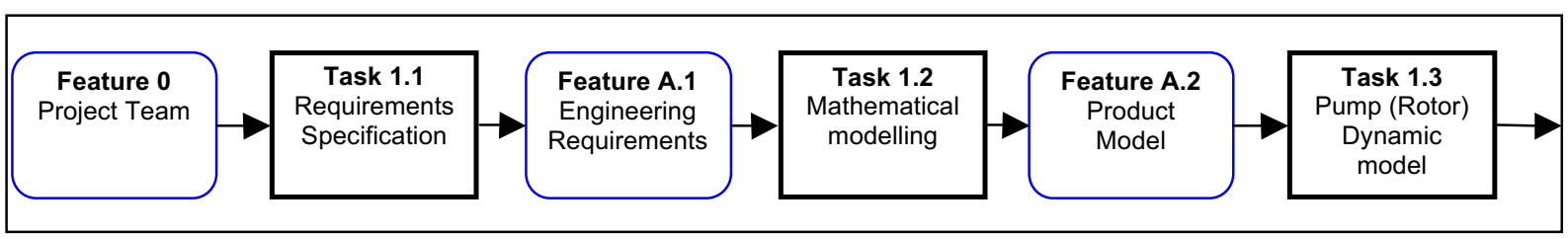

Figure 1: a partial process model representing early design stages

The process model, at this level of detail, is able to perform the process function of task selection. At this stage of the process the precedence relationships are fixed. In later design stages there is a requirement to make an analysis of the current state of the design in order to define the best task to perform next.

\subsection{System features: Information Provision}


A requirement of the system is information provision in the context of task support. This information is intended to support the design process. Experienced designers and engineers will not seek out this supporting information since during the early stages of system development they are very likely to be the source of that information. Catalogue type information (standard dowels, bolts, bearing sizes, etc) will be provided in the task pages, through links to the catalogue. An example of the information provision is shown in Figure 2. This is a section of the task page, which contains a description of how to carry out the task - in this case, bearing selection.

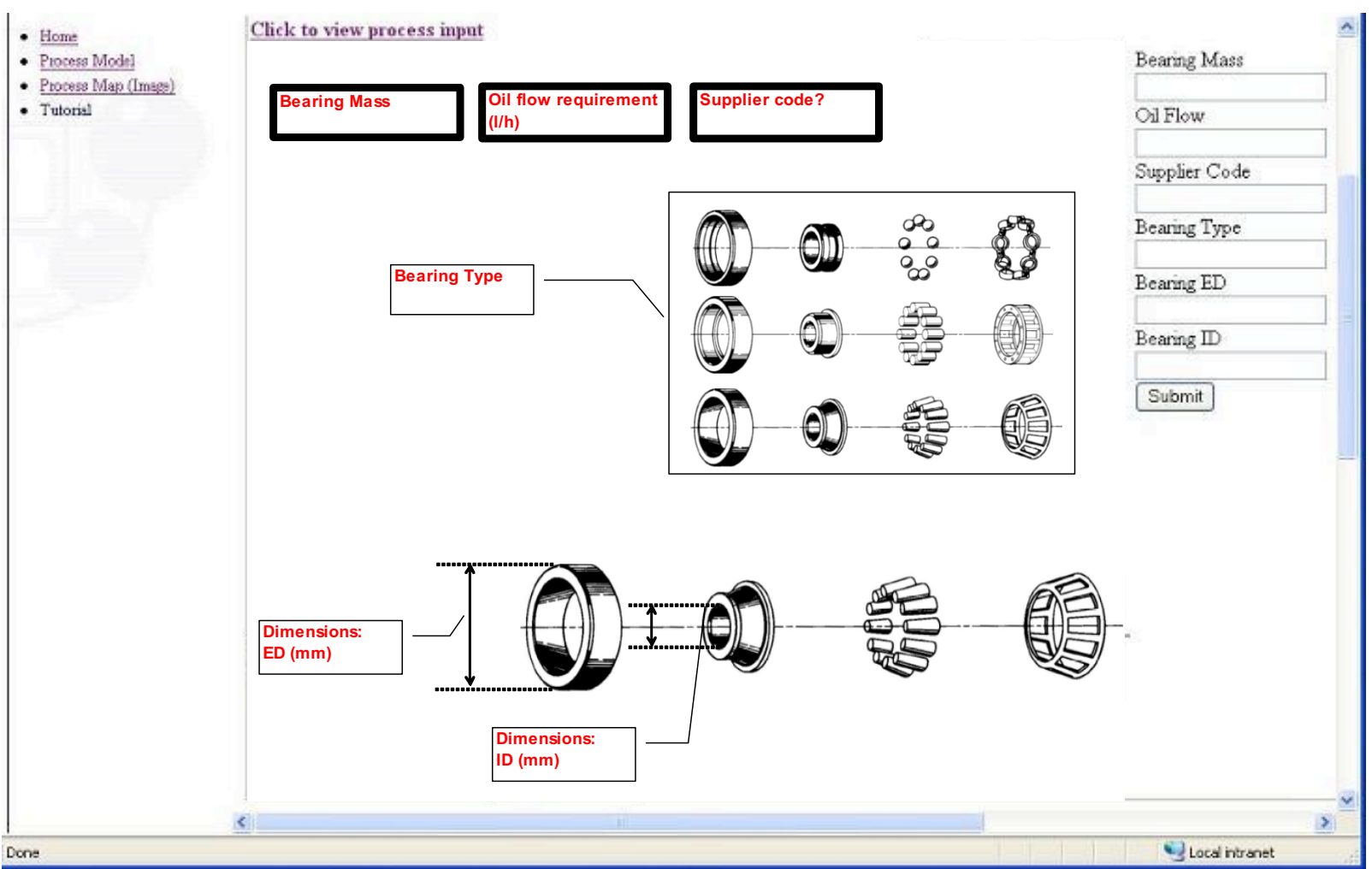

Figure 2: Screen shot of bearing design task page.

To support the development of the product in terms of functionality, it was considered useful to display examples of previous solutions to similar problems to provide examples of how the problem was solved. Images are one method to show previous solutions. Another method is access to previous project information: in this case, a hyperlink to the project archive directory, which includes original requirements documents, test reports and so on.

At later stages of the design process a high level of detail is necessary. Additional information provided to the designers in these stages includes, in addition to the task description: guidelines, links to catalogues and internal stores for standard parts (as well as guidance on what parts are used), and contact details for specialists. The use of standard parts is something that is relatively easy to apply if the information is already available in an electronic format. The use of non-standard parts increases cost and complicates supply.

What emerged as one of the most important supporting information elements was the use of component images on component specification pages. 3-D renders of previously used components are instantly recognised by the engineers and designers, and the features used on these components can be seen, prompting discussion and thought about the rationale that led to the use of those features, as well as alternative solutions that were considered and abandoned. Although this only applies to experienced designers, that richness of information is lost with a menu driven, text based 'previous solutions' option on the page. This became apparent only when images were introduced - on previous versions of the system they were not specifically requested, it was only when the Web based version was developed it became easy to include images on the pages. 


\subsection{System features: Project Tracking and Data Provision}

One of the key functions that the system performs is support for a parameterised product data model. This model can be applied to design (of variant products) from the early stages. If we assume that within a variant design the major mechanical components will be similar, design (for function) is essentially a scaling problem. The second major influence is design to cost. This becomes a selection problem (selection of a component, method, or value). The parameterised product model supports these efforts by assigning and tracking parameter values that are required by subsequent design stages. The result in terms of functionality and cost performance is assessed by the design team. To aid these decisions, the source of the inputs or constraints can be identified.

In the initial knowledge capture exercise, a conceptual model of the pump was developed. Figure 3 shows a part of the model. This was developed as a means to capture and represent data about the product during the design process. Through creating slots for each of the objects (this parametric data model was displayed using protégé 2000) the product parameters and data structure could be shown. This provided a visual tool for validation of the parametric product data model. This data structure was then transferred to the MySQL database, with a PhP interface enabling data input from the task pages.

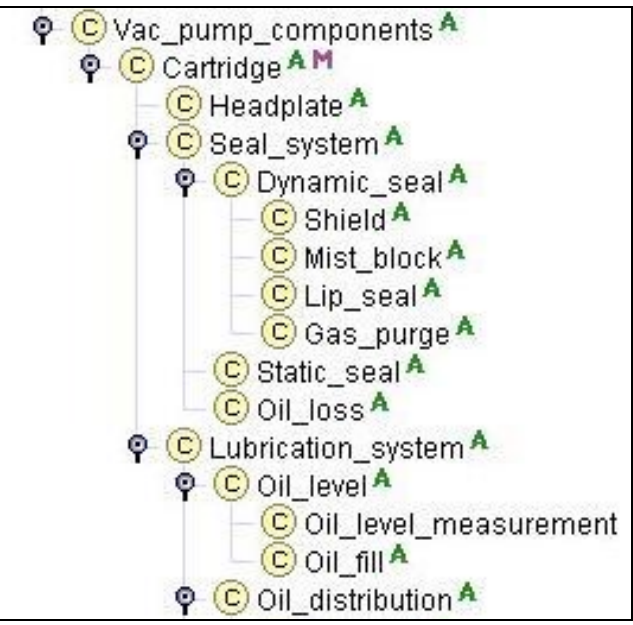

Figure 3: partial parametric data model

The development of the process model highlighted the need for an additional parameter set that sits outside of the product data model. There are several instances in which the data required by a design task is not directly related to the product structure: requirements, function, and performance are not included. There are also task parameters that represent people (project team members), organisations (customers) and data relating to the product in service, such as process type, environment, country (which influences power supply data and documentation language). These additional data elements are represented in the process features, and have become part of the product parametric data model. Figure 4 shows the engineering requirements feature. Several of these parameters are not related to the product structure. This highlights a unique element of a close integration of the design process with the design knowledge reuse method. Developing the design data model in isolation resulted in a description of the product structure. Developing the data model alongside the development process showed the relationship that exists between product geometry and non-structural parameters, and the fact that both are required for a complete representation. 


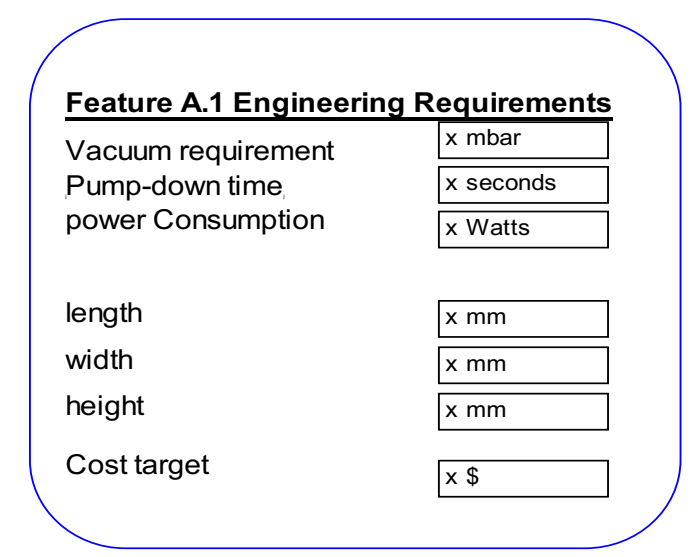

Figure 4: Engineering Requirements Feature

As the process model developed, relationships between the product parameters become apparent: the source of particular product parameters (i.e. the task in which they are defined) becomes apparent. Tracking back through the task model shows those tasks in which critical parameters are defined (tasks with multiple dependencies), as well as tasks which are the source of constraints. During the development of the system, the engineers expressed a preference for visibility of design parameters. That is, the system should show where the parameter was defined. In terms of system design, this can be achieved through a clickable interface such that the user can click a link for each input parameter to view the task in which it was defined. This provides useful context in deciding whether the constraint warrants a change of the original parameter.

The next version of the system could also include a rationale module to describe why particular values are assigned to parameters, and by whom. This could help to support trade-off decisions. Another method to support these decisions is knowledge based support.

\subsection{System features: Knowledge Based Support}

During the design process, the selection of a value for each parameter impacts on the potential range of subsequent parameters (and in some cases previous parameters). The relationship can be direct, such as: the outer diameter of a bearing defines the size of the bore required to house that bearing. In other cases the relationship is not direct, particularly where the parameters are non-geometrical. For example, the required throughput of a vacuum pump influences the most appropriate inlet bore diameter. Throughout the product model there are related parameters. The nature of the relationship is well known in some cases. These relationships can be captured, and a knowledge based approach applied to providing task support or automation.

In the current system, the knowledge based support is provided through the code embedded in active web pages. Parameters are retrieved from the database and calculations made. This is currently achieved through apache, MySQL, and php. The technology applied to the knowledge based support is appropriate for the test system, however a larger scale implementation may require a specific or more comprehensive knowledge expression language such as KIF, or ontology based methods such as OWL. The interface for adding knowledge to the system will also have to be improved. 


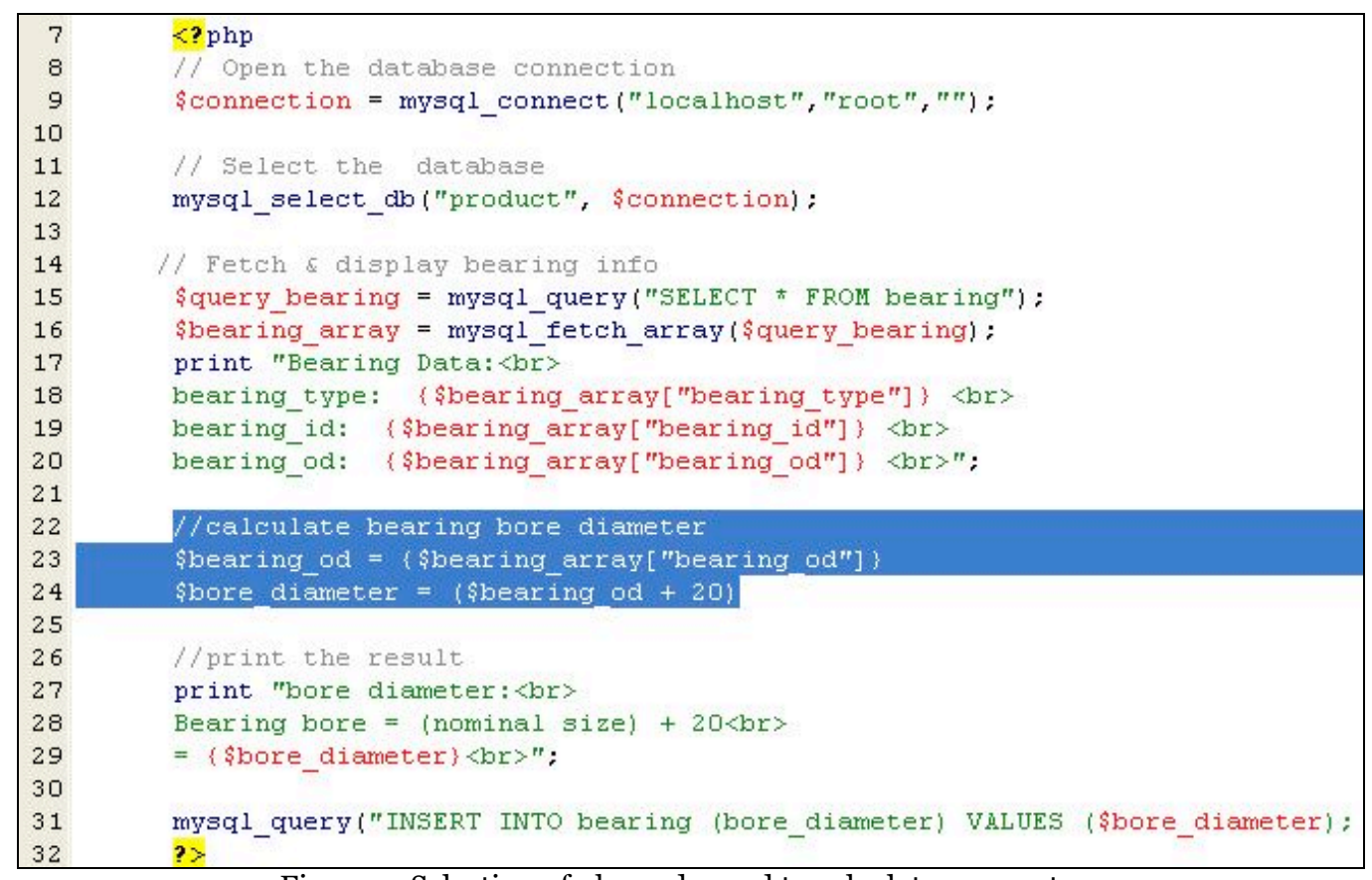

Figure 5: Selection of php code used to calculate parameters

The example in Figure 5 shows the most simple knowledge based support. A product parameter (bearing OD) that was entered on an earlier task page has been retrieved from the database. The bearing bore should be 20 microns larger than the bearing OD. The highlighted text makes this calculation (\$bearing_od + 20) then saves the result in the database. More complex calculations can be made using the same method.

Difficulty arises from three main areas: knowledge value vs. effort, complex interrelationships and step change.

The effort required to embed knowledge based support and automation is high. The degree of effort required for capture does not always correspond to the value of that knowledge. The value of the knowledge is also difficult to define. As such, the need to justify the effort in commercial terms may result in knowledge capture being abandoned.

There are complex interrelationships between parameters in the product model. Changing one parameter value has far reaching effects. Whilst it is possible to calculate the effects of change, this may require extensive effort, particularly where the degree of automation is low. Where there are many parameters which are related, the aim is to design an optimum solution based on the product requirements. Optimisation problems for several interrelated parameters require more effort than knowledge capture for less complex automation.

Step changes in product parameters are considered throughout the design process. Examples of step changes are manufacturing method selection, mechanism type selection, and component type selection. For each step change there may be an impact on earlier values. These feedback loops are built into the process model, however providing knowledge based support to the product model is complex.

\section{DEVELOPING THE INTERFACE}

The interface of the system is flexible, since it is simply a representation of the data layer. A variety of representations are possible for the data and process model. The development of the interface has proved to be important not only in terms of system usability and understanding, but also in capturing the important elements of the system. Providing an interface for the system focused the discussion on comments about the desired functionality of the system. Because the capability of the system is visible through a working prototype, further details about how that capability would be useful in practice were found. The richness of knowledge about the system was much greater when the system was more developed. The current version uses Web pages to show the task as the primary representation. The input data is provided on the task page, and a form to enter the data which 
the task should create. Supporting information is also provided as part of the task page, however to limit the task to a single page this is kept to a minimum, with hyperlinks to the more extensive descriptions. Images of the task are presented where this is appropriate. There is a representation of the process (showing the previous task, data source, and next task(s)), which is currently static but in future versions these process objects will be fully interactive: the user can click to view the next or previous task, or more details about data input. The capability of php to retrieve data from a database, perform calculations on it, and display it on the page, is ideal for this type of developmental system. It is open source, with several open source IDEs and a wide range of resources available, so it represents a highly accessible technology.

\section{SYSTEM EVALUATION AND FUTURE DEVELOPMENT}

System evaluation took place through ongoing evaluation, including validation of process models and data models by the participants, as well as a series of workshops to evaluate the prototype system. Most of the engineers and designers that took part in the workshops were also involved with the original knowledge capture exercise. The workshops were carried out at two stages: the first version, an Excel based model, was assessed. The second version, a Web based model, incorporated comments and changes requested from the original workshop.

The evaluation of the system prototype showed that the value of this information is lower in the early stages of the process. What is useful at the early stages is the application of a data model to the process, which shows the parameters required by a task, their source, and a description of the output (parameters that are required in order for subsequent tasks to progress). If an engineer can see the source of data and understand where it came from, the implications of change are clearer. If the (data) requirements of the next task are known, then time need not be wasted specifying other design parameters that are not directly required.

It was very interesting to note that the addition of graphics to the interface brought a great deal of value. The graphics prompted discussion about the component, including design function trade-offs, manufacturing methods, rejected solutions, and issues with non-local trade-offs (decisions taken for components which are part of a modular system). Where just the parameters and a textual task description were shown (as with previous versions of the system) the discussion remained relatively narrow, focusing on the task itself and the likely output. The addition of graphics appeared to prompt a more detailed thought process about the many influences on the component.

It was found that only a few important parameters lead the design of the product. By focusing on the minimum parameter set to generate a working concept model, constraints are minimised and time can be saved.

It was proposed by the engineers that the next logical step in the development of the system would be to create parametric component models in the CAD systems, and provide an input to them through the parametric product model. This would reduce the effort required in the embodiment and detail phases.

Project monitoring is not currently part of the process model. Methods such as ISO900o, QFD, FMEA, the commercial stage gate process, Six Sigma or any such quality tool could potentially be integrated with the system. It is felt that the addition of such tools could reduce the perceived value of the tool for engineers and designers. It may be considered useful by project managers and other commercial functions. The ability to separate the data, logic and presentation layers enables the addition of functionality without additional burden on all representations. Views could be customised for different functions.

Workflow modelling could increase the efficiency of the process based system. Automatic notifications, task assignment and workload optimisation could reduce possible waiting time in the system where strong precedence links exist. This type of system could also aid real time critical path analysis and project monitoring.

\section{CONCLUSIONS}

The application of the process model along with automation of simple calculations was considered beneficial to the product development process. The system can help engineers and designers focus their effort on the most appropriate tasks. The development of the parametric product data model allows the engineers to focus on those design parameters which have most influence on product performance during the early stages of product development. 
By storing this parameter set alongside the process model, engineers can access the current project state (from remote locations since it is based on Web enabled technologies) and have a view of the previous decisions which constrain the current task. The supporting information provided alongside the task model supports the tasks.

By providing a single product data model and linking it to design tasks, the management of the design project becomes more transparent.

\section{ACKNOWLEDGEMENTS}

The authors would like to acknowledge the member of the research project team at Loughborough University for their continued professional guidance and support throughout the project: Keith Case, Jenny Harding, Bob Young, Shilpa Dani and Sean Cochrane. The authors would also like to acknowledge the financial support of the EPSRC through the Cranfield University IMRC.

\section{REFERENCES}

[1] Sivaloganathan, S. and Shahin, T.M.M. (1999) Design reuse: an overview. Proceedings of the Institution of Mechanical Engineers - Part B - Engineering Manufacture 213, 641-655.

[2] Busby, J.S., 1999, The Problem with Design Reuse: An Investigation into Outcomes and Antecedents, Journal of Engineering Design [10] 277-297.

[3] Ullman, D.G. (2002) Toward the ideal mechanical engineering design support system. Research in Engineering Design 13, 55-64.

[4] Baxter, D. and Gao, J. (2005) Formalising Customer Requirements as an Input to a Design Knowledge Reuse System. In: Proceedings of the Third International Conference on Manufacturing Research (ICMR2005), 6-8 September 2005, Cranfield University, UK.

[5] Chakrabarti, A., Morgenstern, S. and Knaab, H. (2004) Identification and application of requirements and their impact on the design process: a protocol study. Research in engineering design 15, 22-39.

[6] Ullman, D.G. (2003) The Mechanical Design Process. McGraw-Hill, London.

[7] Pahl, G. and Beitz, W. (1988) Engineering Design: a systematic approach. The Design Council, London.

[8] Gardam, A. and Burge, S.E. (1997) Changes in the Engineering Design Process. In: Advances in Maufacturing Technology XI, 9-11 September 1997, Glasgow Caledonian University.

[9] Mcdermott, Handfield, 200o. Concurrent development and strategic outsourcing: Do the rules change in breakthrough innovation?, The Journal of High Technology Management Research 11 35-57.

[10] Finger, S. (1998) Design reuse and design research - keynote paper. In: proceedings of the Engineering Design Conference '98, 23-25 June 1998, Brunel University, UK.

[11] Clarkson, P.J. and Hamilton, J.R. (2000) Signposting, A Parameter-driven Task-based Model of the Design Process. Research in Engineering Design 12, 18-38.

[12] Park H; Cutkosky MR (1999). Framework for modeling dependencies in collaborative engineering processes. Research in engineering design 11: 84-102 